\title{
Brain-to-Blood Transporters for Endogenous Substrates and Xenobiotics at the Blood-Brain Barrier: An Overview of Biology and Methodology
}

\author{
Tetsuya Terasaki and Sumio Ohtsuki \\ New Industry Creation Hatchery Center and Graduate School of Pharmaceutical Sciences, Tohoku University, Aoba, Aramaki, \\ Aoba-ku, Sendai 980-8578, Japan; and CREST \& SORST, Japan Science and Technology Agency, Japan
}

\begin{abstract}
Summary: In the past decade, research into P-glycoprotein involving the blood-brain barrier (BBB) has seen a shift in the concept of the BBB as a structural barrier to that of a functional barrier for xenobiotics and changed simultaneously the strategy for the discovery and development of drugs acting in the CNS. As far as making advances in neurotherapeutics are concerned, the brain-to-blood transport function at the BBB will be one of the most important issues. Knowing the limitations of the in vivo and in vitro methods for $\mathrm{BBB}$ efflux research, it is essential to adopt a multidisciplinary approach in investigating the true physiological role of the BBB. Among several methods, the Brain Efflux Index method and the use of conditionally immortalized brain capillary endothelial cell lines, established from transgenic rats harboring temperature-sensitive simian virus 40 large $\mathrm{T}$-antigen gene, are likely to be very useful tools for the $\mathrm{BBB}$ efflux transport research. According to our recent findings
\end{abstract}

using these methods, several transporters in the brain capillary endothelial cells appear to play an important role in reducing the brain level of hydrophilic endogenous substrates produced either in the brain or peripheral organs, e.g., neurotransmitters, neuromodulators, metabolites of neurotransmitters, and uremic toxins. It has been reported also that large hydrophilic molecules, such as IgG, apo-transferrin, and amyloid- $\beta$ peptide, are susceptible to brain-to-blood efflux transport. In the light of the latest findings, we have formed the hypothesis that the $\mathrm{BBB}$ acts as a CNS detoxifying system for both endogenous substrates and xenobiotics in the brain. A fuller understanding of the physiological role of $\mathrm{BBB}$ efflux transporters will provide rational insights to assist in the development of safer neurotherapeutics. Key Words: Blood-brain barrier efflux transport, brain efflux index method, conditionally immortalized cell line, MDR1, ABCG2/BCRP, amyloid peptide.

\section{INTRODUCTION}

Research into the blood-brain barrier (BBB) was initiated in 1885 when Paul Ehrlich gave an intravascular administration of trypan blue to rabbits. However, it took over 80 years to obtain direct evidence that the BBB consists of brain capillary endothelial cells (BCECs), after the electron microscopic studies of Reese, Karnovsky, Brightman, and co-workers. ${ }^{1,1 a}$ In the past 4 decades, the development of several in vivo and in vitro methods has significantly increased the progress in BBB

Address correspondence and reprint requests to Tetsuya Terasaki, Ph.D., Professor, Department of Molecular Biopharmaceutics and Genetics, Graduate School of Pharmaceutical Science, Tohoku University, 6-3 Aoba, Aramaki, Aoba-ku, Sendai 980-8578, Japan. E-mail: terasaki@mail.pharm.tohoku.ac.jp. transport research. These findings include two important physiological roles of the BBB, i.e., 1) to restrict the paracellular permeability of hydrophilic large and small molecules, and 2) to transport several nutrients and proteins to the brain. If the BBB acts only as a structural barrier for blood-borne hydrophilic large and small molecules, simultaneously, the BBB will accumulate the hydrophilic molecules generated in the brain. Several recent studies have revealed that the BBB has multiple transporters involved in the brain-to-blood efflux transport of hydrophilic small molecules generated in the brain, such as neurotransmitters, neuromodulators, end metabolites of neurotransmitters and uremic toxins, and also peptides, such as IgG. Table 1 presents a summary of the brain-to-blood multiple transporters at the BBB and indicates that the BBB has a third important physiological role as a functional barrier, pumping out not only xenobiotics, but also endogenous molecules generated in the brain, into the circulating blood. 
TABLE 1. The Brain-to-Blood Efflux Transport Systems at the Blood-Brain Barrier

\begin{tabular}{|c|c|c|c|}
\hline Type & Substrates & Transporters & Localization \\
\hline \multirow[t]{3}{*}{ Neurotransmitters } & GABA & GAT2/BGT-1 & ND \\
\hline & Serotonin & SERT & A, L \\
\hline & Norepinephrine, dopamine & NET & A \\
\hline Neurotransmitter metabolite & Homovanillic acid & OAT3 & A \\
\hline Excitatory amino acids & L-Glutamic acid, L-aspartic acid & ASCT2, EAATs & A \\
\hline \multirow[t]{2}{*}{ Neuromodulators } & DHEAS, $\mathrm{E}_{1} \mathrm{~S}, \mathrm{E}_{2} 17 \beta \mathrm{G}$ & oatp2 & $\mathrm{A}, \mathrm{L}$ \\
\hline & Glycine, L-alanine, L-proline & ATA2 & ND \\
\hline Uremic toxin & Indoxyl sulfate & OAT3 & A \\
\hline \multirow{7}{*}{ Xenobiotics } & Vincristine, cyclosporine & MDR1/ABCB1 & $\mathrm{L}$ \\
\hline & Estradiol glucuronide & MRP1/ABCC1 & $\mathrm{L}$ \\
\hline & Prazosin, mitoxantron & $\mathrm{ABCG} 2$ & $\mathrm{~L}$ \\
\hline & 6-Mercaptopurine & OAT3 & A \\
\hline & BQ-123 & oatp2 & $\mathrm{A}, \mathrm{L}$ \\
\hline & Azidothymidine, dideoxyinosine & Unknown & \\
\hline & Valproic acid & Unknown & \\
\hline \multirow{3}{*}{ Macromolecules } & Ig & Fc receptor & \\
\hline & Apo-tranferrine & Tf receptor & \\
\hline & $\beta$-Amyloid peptide & LRP-1, MDR1 & \\
\hline
\end{tabular}

EAATs $=$ excitatory amino acid transporters; $\mathrm{E}_{2} 17 \beta \mathrm{G}=$ estradiol-D-17 $\beta$-glucuronide; $\mathrm{Tf}$ receptor $=$ transferrin receptor; $\mathrm{A}=$ abluminal localization; $\mathrm{L}=$ luminal localization; $\mathrm{ND}=$ not determined.

P-glycoprotein $[\mathrm{P}$-gp/multidrug resistance protein 1 (MDR1)], a well-known efflux transport protein of tumor cells, was found in the luminal membrane of the BCECs in 1989 in a series of immunohistochemical studies. ${ }^{1 \mathrm{~b}}$ The active efflux transport function of P-gp at the BBB was demonstrated by means of cultured BCECs. ${ }^{2}$ After the first study of the intravascular administration of ivermectin to the mdr1a knockout mouse by Schinkel et al., ${ }^{3}$ many investigators have confirmed that the P-gp expressed at the BBB plays a very important role in restricting the entry of xenobiotics from the circulating blood into the brain. It can be said that these P-gp research have changed the concept of the BBB as a structural barrier to a functional barrier and also changed the strategies used for the discovery of CNS drugs. Nevertheless, it seems to us that there is a disproportionate emphasis on P-gp function at the BBB. As shown in Table 1, P-gp is only one of many efflux transporters at the BBB.

The progress in $\mathrm{BBB}$ research clearly depends on the development of new methodologies. The best way to study the brain-to-blood transporters at the $\mathrm{BBB}$ in vivo is with the Brain Efflux Index (BEI) method. Then, a way of extending our knowledge of the physiology of BBB efflux transporters is with molecular biological studies performed using conditionally immortalized cell lines as a novel in vitro BBB model. In this review, we shall present an overview of the latest methodology and biology focusing on the brain-to-blood efflux transport system and, then, introduce the hypothetical physiolog- ical role of the BBB efflux transport system as a CNS detoxification system. ${ }^{4}$

\section{IN VIVO BRAIN EFFLUX INDEX METHOD FOR THE ANALYSIS OF BBB EFFLUX TRANSPORT FUNCTION}

There is no perfect single method for investigating the mechanism governing BBB efflux transport, and many different methods have been developed such as the BEI method, distributed model analysis, brain microdialysis method, and BBB efflux transporter gene knockout mice studies. It is very important to understand the limitations of each method, and to select the combination of the methods that are most suited to particular studies. Among these in vivo methods, the BEI method is the most useful method for determining the BBB efflux clearance and clarifying transport function. ${ }^{5}$ The advantages and limitations of the rest of the in vivo methods are described in other review articles. ${ }^{5 a}$

Figure 1A illustrates the principle of the BEI method. The definition of the BEI value is as follows:

$\mathrm{BEI}(\%)=$ (amount of test substrate effluxed at the

$\mathrm{BBB} / \mathrm{amount}$ of test substrate injected into the

$$
\text { brain) } \times 100
$$

To minimize interindividual differences in the amount of test substrate injected, a BBB-impermeable reference compound is injected simultaneously into the brain cortex. Par 2 of the rat cortex was found to be the most 
A. Principle of the Brain Efflux Index method

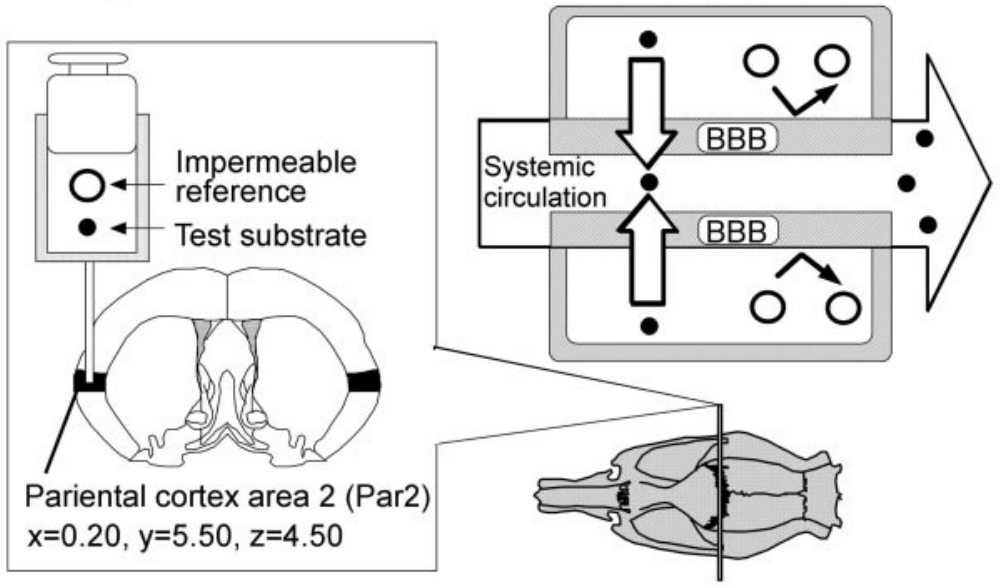

B. Brain-to-blood transport of homovanillic acid

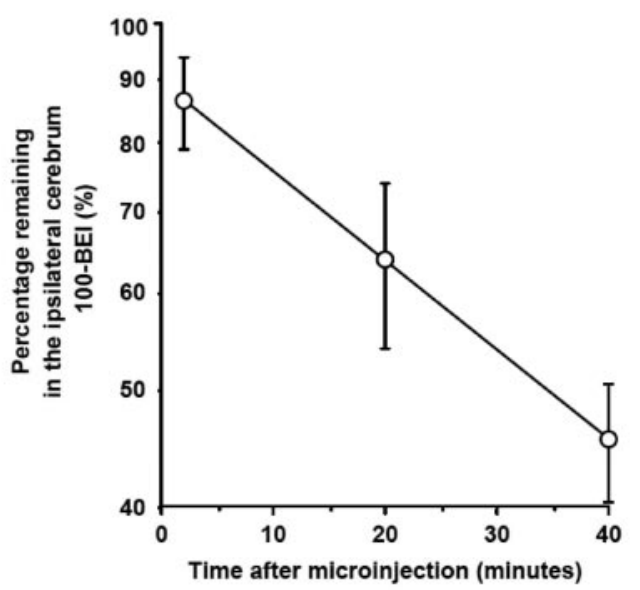

FIG. 1. Principle and a typical example of the BEI method used for the analysis of BBB efflux transport function. Reproduced with permission from (A) Terasaki et al. The brain efflux index method (BEI). In: Alfred Benzon Symposium 45, Brain Barrier Systems (Palson OB, Knudsen GM, Moos T, eds). Copyright (C) 1998, Munksgaad. All rights reserved. ${ }^{53}$ And (B) Mori et al. Rat organic anion transporter 3 (rOAT3) is responsible for brain-to-blood efflux of homovanillic acid at the abluminal membrane of brain capillary endothelial cells. $J$ Cereb Blood Flow Metab 23:432-440. Copyright (C) 2003, Lippincott Williams \& Wilkins. All rights reserved. ${ }^{6}$

suitable region for microinjection. ${ }^{5}$ The following equation is used to determine the percentage of the test substrate remaining in the ipsilateral cerebrum:

$$
\begin{aligned}
100 & -\operatorname{BEI}(\%) \\
& =\left[1-\left(\mathrm{X}_{\text {brain,test }} / \mathrm{X}_{\text {brain,ref }}\right) /\left(\mathrm{C}_{\text {inject,test }} / \mathrm{C}_{\text {inject,ref }}\right)\right] \times 100
\end{aligned}
$$

where $\mathrm{X}_{\text {brain,test }}, \mathrm{X}_{\text {brain,ref }}, \mathrm{C}_{\text {inject,test, }}$ and $\mathrm{C}_{\text {inject,ref }}$ represent the amount of test substrate and reference compound in the ipsilateral cerebrum and the concentration of test substrate and reference compound in the injectate, respectively. The BBB efflux rate constant, $\mathrm{K}_{\mathrm{eff}}$, can be obtained from the slope of the semilogarithmic plot of the value of (100-BEI) versus time. Figure 1B illustrates a typical efflux curve generated by the BEI method. ${ }^{6}$ The BBB efflux clearance ( $\left.\mathrm{CL}_{\mathrm{BBB}, \text { eff }}\right)$ is determined by equation 3:

$$
\mathrm{CL}_{\mathrm{BBB}, \mathrm{eff}}=\mathrm{K}_{\mathrm{eff}} \times \mathrm{V}_{\text {brain,test }}
$$

where $V_{\text {brain,test }}$ represents the distribution volume of the test substrate in the cerebrum and can be determined by the brain slice uptake study. For concentration-dependent studies, the following equation can be used,

$$
\begin{gathered}
\mathrm{J}_{\mathrm{BBB}, \text { eff }}=\mathrm{CL}_{\mathrm{BBB}, \text { eff }} \times \mathrm{C}_{\text {inject,test }}=\mathrm{V}_{\max } /\left(\mathrm{K}_{\mathrm{m}}+\mathrm{C}_{\text {inject, test }}\right) \\
+\mathrm{CL}_{\text {non }} \times \mathrm{C}_{\text {inject,test }}
\end{gathered}
$$

where $\mathrm{J}_{\mathrm{BBB}, \mathrm{eff}}, \mathrm{V}_{\text {max }}, \mathrm{K}_{\mathrm{m}}$, and $\mathrm{CL}_{\text {non }}$ represent the $\mathrm{BBB}$ efflux transport rate, the maximum $\mathrm{BBB}$ efflux transport rate, the Michaelis constant and the nonsaturable $\mathrm{BBB}$ efflux clearance, respectively. Instead of the $C_{\text {inject,est }}$ in equation 4 , the cerebrum concentration of test substrate injected, $\mathrm{C}_{\text {brain,test }}$ can be used to obtain the $\mathrm{K}_{\mathrm{m}}{ }^{\prime}$ value, reflecting the brain concentration of the test substrate. $\mathrm{C}_{\text {brain,test }}$ is estimated from the $\mathrm{C}_{\text {inject,test }}$ divided by the dilution factor of the injectate in the cerebrum, i.e., $\sim 30$ fold. $^{5}$

The $\mathrm{CL}_{\mathrm{BBB} \text {,eff }}$ of [ $\left.{ }^{3} \mathrm{H}\right] 3-\mathrm{O}$-methyl-D-glucose (3-OMG), a substrate of the facilitated transport system for hexose (GLUT1) in BCECs, was determined by the BEI method as $102 \pm 16 \mu \mathrm{l} /(\mathrm{min} \cdot \mathrm{g}$ brain) and was found to be similar to the BBB influx clearance $\left(\mathrm{CL}_{\mathrm{BBB}, \text { inf }}\right)$, i.e., $93 \pm 14$ $\mu \mathrm{l} /(\mathrm{min} \cdot \mathrm{g}$ brain). These results demonstrate that the BEI method is valid for the analysis of the symmetrical transport mechanism at the BBB in a quantitative manner. ${ }^{5}$ In other words, the BEI method can analyze the brain-toblood efflux transport system at the BBB by comparing the $\mathrm{CL}_{\mathrm{BBB} \text {,eff }}$ and $C L_{\mathrm{BBB} \text {,inf }}$. As shown in a later part of this review, several important physiological functions of the BBB efflux mechanisms have been elucidated by the BEI method. One of the advantages of the BEI method is that it enables the direct measurement of the brain-toblood elimination rate of the test substrate. The limitations of the BEI method are that 1) it is difficult to identify the transport process either in the abluminal membrane or luminal membrane of the BCECs, and 2) we cannot use the BEI method for substrates that are significantly metabolized in the cerebrum.

\section{IN VITRO METHOD TO IDENTIFY THE BBB EFFLUX TRANSPORTER PROTEIN AND CLARIFY THE PHYSIOLOGICAL ROLE}

There are some limitations associated with the above in vivo methods for identifying BBB efflux transporter 
A. Cellular uptake study Single culture

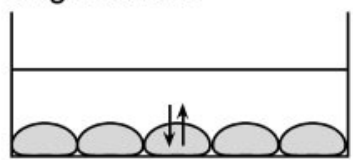

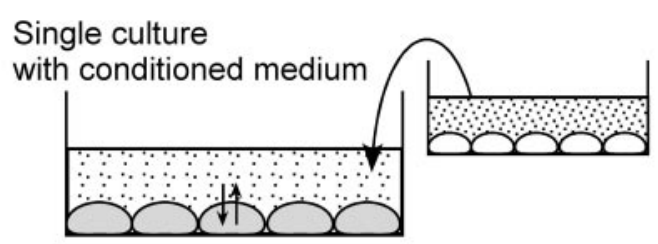

B. Transcellular transport study

Single culture

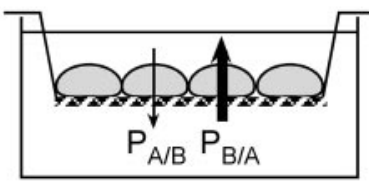

TR-BBB cells
Contact co-culture

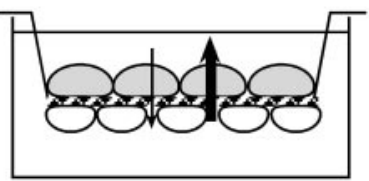

TR-AST and/or TR-PCT cells
Non-contact co-culture

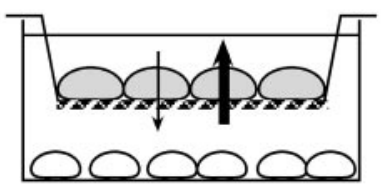

C. Establishing conditionally immortalized cell lines

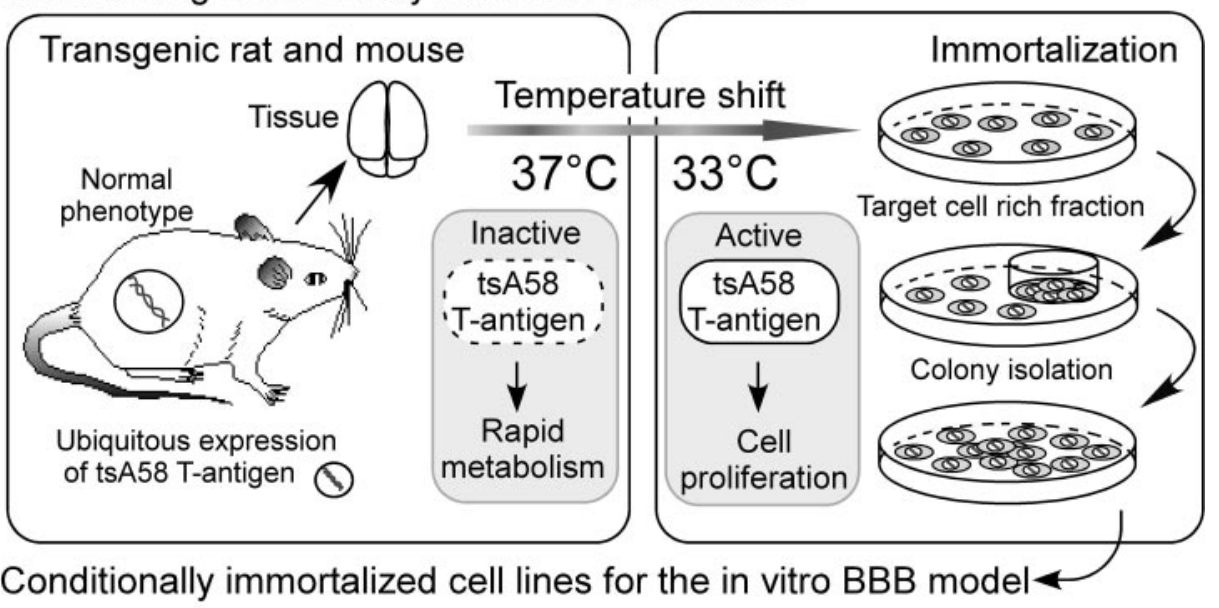

D. Conditionally immortalized cell lines for the in vitro BBB model

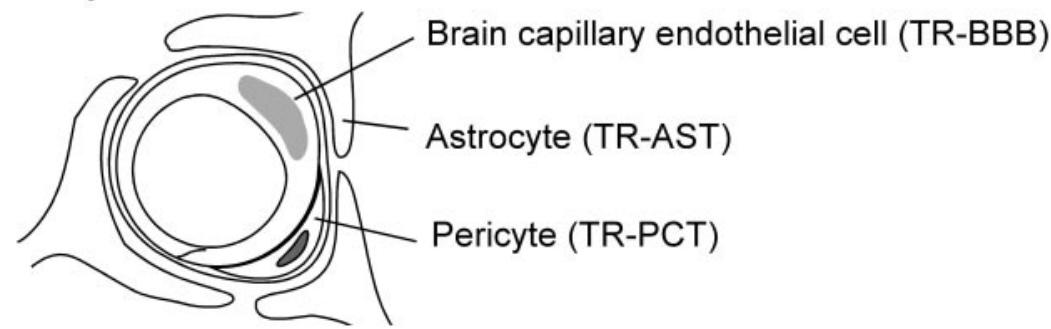

FIG. 2. Establishment and application of conditionally immortalized cell lines as in vitro BBB models.

molecules, although they can provide important information about how the brain-to-blood transport pathway contributes significantly to CNS distribution. The in vitro BBB method is useful for identifying a transport inhibitor and clarifying the transport mechanism at the plasma membrane of BCECs. Moreover, it can provide valuable information to support drug discovery research and also to determine the rank order for the BBB permeability of candidate compounds. Three types of in vitro BBB model can be employed, i.e., 1) cultured BCECs, 2) freshly isolated BCECs, and 3) plasma membrane vesicle of BCECs.
Primary culture and immortalized cell lines of brain capillary endothelial cells

Many culture systems of BCECs have been developed, e.g., primary cultures of mouse, rat, or cow, or immortalized cell lines of mouse, rat, cow, pig, or human. ${ }^{7}$ If the paracellular permeability rate is not significantly low, either steady-state cellular uptake studies or the cell washout studies can be used to analyze the efflux mechanism from the confluent cell monolayer (FIG. 2A). If the tight junction limits the paracellular permeability, transcellular transport studies can be used to determine the Basal-to-Apical permeability $\left(\mathrm{PS}_{\mathrm{B} / \mathrm{A}}\right)$ and the Apical- 
to-Basal permeability ( $\mathrm{PS}_{\mathrm{A} / \mathrm{B}}$ ) (FIG. 2B). The ratio of $\left(\mathrm{PS}_{\mathrm{B} / \mathrm{A}}\right)$ and $\left(\mathrm{PS}_{\mathrm{A} / \mathrm{B}}\right)$ are useful parameters for evaluating brain-to-blood efflux transport at the BBB. One of the limitations of the primary culture system is the large variation in the transport rate of different isolates. Therefore, large amounts of cells need to be stored from the same batch preparation. The transporter genes of both primary culture and immortalized cell lines appear to be downregulated, and it is possible to underestimate the transport activities using the single culture system.

As illustrated in Figure 2, A and B, several different types of coculture system have been developed. Compared with the single culture system, the coculture system of primary BCECs and astrocyte cells have been reported to provide greater tight junctions ${ }^{8}$ and a higher expression of P-gp. ${ }^{9}$ This is useful for transcellular transport studies.

\section{Conditionally immortalized cell lines}

Recently, we have established conditionally immortalized brain capillary endothelial cell lines from temperature sensitive simian virus 40 (SV40) large T-antigen gene transgenic mice and rats, TM-BBB ${ }^{10}$ and TR$\mathrm{BBB},{ }^{11}$ respectively. Figure $2 \mathrm{C}$ illustrates the principle of the method to establish the conditionally immortalized cell lines. Transgenic mice/rats express mutant virus gene product, tsA58 SV40 large T-antigen, which is inactive at $37^{\circ} \mathrm{C}$ and metabolized rapidly, but is active at $33^{\circ} \mathrm{C}$. Therefore, the established cell line needs to be cultured at $33^{\circ} \mathrm{C}$, whereas the transport studies can be performed at $37^{\circ} \mathrm{C}$. There is good agreement between the in vitro uptake rate determined using TM-BBB or TR$\mathrm{BBB}$ cells and the in vivo $\mathrm{BBB}$ transport rate for the model substrates to be transported by the carrier-mediated system. ${ }^{7}$ This shows that the transporter gene may not be significantly downregulated in these cell lines, although the expression of the tight junction proteins and P-gp are low. Based on the same principle (FIG. 2C), several BBB-related cell lines have also been established from transgenic rats, i.e., astrocytes (TR-AST) and pericytes (TR-PCT) (FIG. 2D). Because the tight junction protein $^{12}$ and transporter genes ${ }^{13}$ of TR-BBB cells are induced by the conditioned medium of these cell lines, the coculture system represents a useful and reproducible in vitro BBB model.

\section{Isolated brain capillary}

Isolated brain capillaries have been used for in vitro transport studies. ${ }^{14}$ Because the expression of transporter proteins of the isolated brain capillaries is the same as those in vivo, we can confirm the expression of transporter protein at the BBB when the purity is high. There are some limitations in characterizing the active efflux transport because of the low level of ATP in the isolated cells.

\section{Plasma membrane vesicles of brain capillary endothelial cells}

Luminal and abluminal membrane vesicles of BCECs are also used for the functional and biochemical characterization of BBB efflux transporters. There are some limitations for the lipophilic substrates to minimize nonspecific adsorption to the filters used for separation. EAAT $^{15}$ and system $\mathrm{A}^{16}$ have been studied using membrane vesicles.

\section{Tansporter gene expressing system}

P-gp is the most important efflux transporter at the $\mathrm{BBB}$. In drug discovery research, it is very important to know the extent of human BBB permeability. Because a species difference in transport function between human MDR1 and mouse mdr1a has been reported, ${ }^{17}$ it is better to use human MDR1 expressing cells for CNS drug discovery. A fairly good correlation has been reported between the in vivo brain concentration ratios of mdr1a $(-/-)$ to $(+/+) \mathrm{CF}-1$ mice and the in vitro transcellular transport ratios $\left(\mathrm{PS}_{\mathrm{B} / \mathrm{A}}\right) /\left(\mathrm{PS}_{\mathrm{A} / \mathrm{B}}\right)$ from mdr1a gene-transfected cells. These results suggest that the in vitro method is valid for evaluation of the P-gp contribution as far as in vivo BBB efflux is concerned. Moreover, the human MDR1 expressing system is useful for reliably predicting the in vivo relevance of P-gp in humans. ${ }^{17}$

If there is a selective and significant inhibitor of the known BBB efflux transport system, such as benzylpenicillin for organic anion transporter 3 (OAT3), the BEI method would be useful for evaluating the contribution of candidate transport systems. ${ }^{6}$ Then, the transporter gene expression system, such as a stable or transient transfectant cell line of cDNA, or cRNA injected Xenopus oocytes, is a useful method for obtaining direct evidence that the test compound is a substrate of the transporter gene product.

\section{MOLECULAR BIOLOGY AND PHYSIOLOGY OF THE BRAIN-TO-BLOOD TRANSPORT SYSTEM AT THE BBB}

\section{The BBB efflux transport of neurotransmitters}

The function of the BBB used to be considered as the retention of neurotransmitters in the brain. Nevertheless, our recent studies have shown that neurotransmitters undergo efflux from the brain across the BBB. ${ }^{18,19}$ Therefore, the physiological role of the BBB is not merely as a structural barrier, it also acts as a functional barrier regulating the turnover of endogenous substrates in the brain (FIG. 3).

GABA, which is a suppressive neurotransmitter, is widely distributed throughout the CNS. Using the BEI method, we have shown that $\left[{ }^{3} \mathrm{H}\right] \mathrm{GABA}$ is eliminated from brain. ${ }^{18}$ Betaine/GABA transporter-1 (BGT-1; SLC6A12), which corresponds to GABA transporter 2 


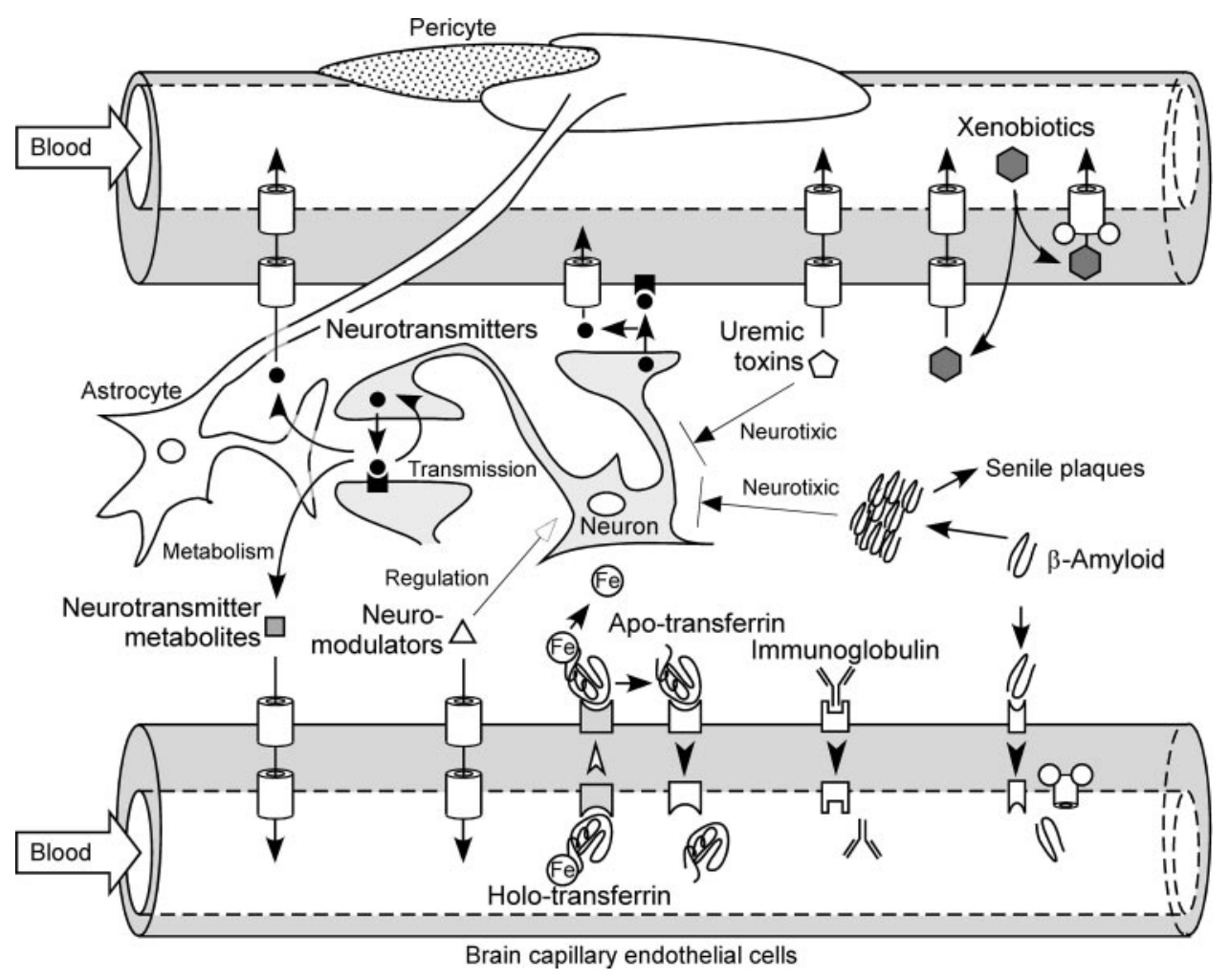

FIG. 3. Hypothetical physiological role of the brain-to-blood efflux transport systems at the blood-brain barrier.

(GAT2) in the mouse, is localized at the BCECs and is involved in the efflux transport of GABA at the $\mathrm{BBB}^{20}$ (Table 1). Neurons and astrocytes express GAT1 and 3, indicating that the GABA transporters expressed at the $\mathrm{BBB}$ are different from those expressed by neurons and astrocytes. Indeed, the efflux rate of $\left[{ }^{3} \mathrm{H}\right] \mathrm{GABA}$ across the BBB was not inhibited, but increased by nipecotic acid, which is a specific inhibitor of neuronal and glial GABA transporters. ${ }^{18}$

BCECs also exhibit monoamine uptake activity ${ }^{21}$ (FIG. 3). Immunohistochemical analysis showed that norepinephrine transporter (NET) is localized at the abluminal membrane of mouse BCECs, and serotonin transporter (SERT) is localized at both the abluminal and luminal membranes ${ }^{22}$ (Table 1). The brain microvasculature is thought to be regulated by monoamines released from adrenergic and serotonergic neurons. Therefore, the abluminally localized NET and SERT would function as an inactivation system for neurotransmitters around the brain capillaries. Serotonin is a potent vasoconstrictor, and serotonin secreted from platelets enhances blood coagulation. Based on these pharmacological functions, one possible function of the luminally localized SERT is clearance of serotonin from the cerebral intravascular space to maintain cerebral blood flow.

\section{The BBB efflux transport of amino acids}

L-Asp is an excitatory amino acid as well as L-glutamic acid (L-Glu), whereas D-aspartic acid (D-Asp) functions as a precursor of NMDA and also influences the secretion of several hormones, such as growth and luteinizing hormones, testosterone, melatonin, and oxytocin. The BBB exhibits stereo-selective efflux transport of Asp, transporting the L-isomer but not the D-isomer. ${ }^{23}$ This L-isomer-selective Asp efflux transport is consistent with the CNS function of each isomer of Asp because the accumulation of L-Asp leads to excitatory neurotoxicity, whereas D-Asp needs to be stored as a precursor of NMDA. System ASC transporter, ASCT2, mediates L-isomer selective transport and is localized at the abluminal membrane of BCECs $^{19}$ (Table 1). Excitatory amino acid transporters, EAATs, have also been detected in the abluminal membrane fraction of BCECs, ${ }^{15}$ whereas they transport both isomers of Asp. Therefore, ASCT2 is likely to be mainly involved in the L-isomerselective efflux transport of Asp at the BBB, although the possibility that the D-Asp efflux transport process may not be present on the luminal side cannot be ruled out.

System A is a transport system for small neutral amino acids that accepts L-Ala, L-proline (L-Pro), glycine (Gly), and is present on the abluminal membrane of BCECs. ${ }^{16,24}$ Of the three isoforms of system A transporter, ATA2 mRNA was present in 93- and 2140-fold greater concentrations than ATA1 and ATA3 in the mRNAs in TR-BBB cells, respectively. ${ }^{25}$ This result suggests that ATA2 is responsible for system A efflux transport at the BBB (Table 1). Under hypertonic con- 
ditions, ATA2 mRNA in TR-BBB cells is induced concomitantly with activation of the transport function. ${ }^{25}$ Osmoregulation in the brain is important for maintaining a constant milieu in the CNS. Therefore, osmoregulation of ATA2 may contribute to the regulation of the osmolarity in the brain and the cell volume in BCECs.

\section{The BBB efflux transport of neurotransmitter metabolites}

Homovanillic acid (HVA) is a major metabolite of dopamine. The HVA concentration in the brain is increased when probenecid or octanoic acid is administered peripherally, ${ }^{26,27}$ and the brain-to-blood efflux transport rate of HVA is faster than the blood-to-brain HVA influx rate across the BBB. ${ }^{28}$ These results suggest that a probenecid- and octanoic acid-sensitive brain-toblood efflux transport system functions as the clearance system for HVA (FIG. 3). The brain-to-blood efflux transport rate of HVA is significantly inhibited by benzylpenicillin, which is an OAT3-selective inhibitor, ${ }^{29,30}$ and OAT3 is localized at the abluminal membrane of the BCECs. ${ }^{6}$ This suggests that OAT3 is the transporter responsible for the brain-to-blood efflux of HVA (Table 1). The metabolites of monoamine neurotransmitters include many organic anions. The HVA transport by OAT3 is inhibited by various anionic metabolites of neurotransmitters, such as 3,4-dihydroxyphenylacetic acid derived from dopamine, vanillylmandelic acid, 3,4-dihydroxymandelic acid, and 4-hydroxy-3-methoxyphenylglycol derived from norepinephrine and epinephrine, 5-hydroxyindole acetic acid and 5-methoxytryptophol derived from serotonin, and imidazole-4-acetic acid and 1-methyl-4-imidazolic acid derived from histamine. In contrast, neurotransmitters themselves, such as dopamine, norepinephrine, serotonin, and histamine, do not inhibit this activity. Although compounds that have inhibitory activity toward a transporter are not always substrates, the above result raises the possibility that OAT3 mediates the BBB efflux transport of various neurotransmitter metabolites.

\section{The BBB efflux transport of neurosteroids}

Dehydroepiandrosterone sulfate (DHEAS) is a neurosteroid that can interact with GABA type A receptors and $\sigma$ receptors to increase memory and learning ability and to protect neurons against excitatory amino acidinduced neurotoxicity. Using the BEI method, $\left[{ }^{3} \mathrm{H}\right] \mathrm{D}$ HEAS has been shown to be eliminated from the brain to the circulating blood across the $\mathrm{BBB}^{31}$ (FIG. 3). TM-BBB cells exhibit uptake of $\left[{ }^{3} \mathrm{H}\right] \mathrm{DHEAS}$, and this process is significantly inhibited by an organic anion transporting polypeptide 2 (oatp2)-selective substrate, digoxin. ${ }^{31}$ Oatp2 is localized on both the luminal and abluminal membrane of rat BCECs. ${ }^{32}$ Thus, oatp2 expressed at the BBB plays a role in the BBB efflux transport of DHEAS (Table 1).
Estrone-3-sulfate $\left(E_{1} S\right)$ is also a neurosteroid and one of the substrates of oatp2. Using the BEI method, $\mathrm{E}_{1} \mathrm{~S}$ was found to be eliminated from brain to the circulating blood across the BBB. ${ }^{33} \mathrm{E}_{1} \mathrm{~S}$ efflux transport is a saturable process and is inhibited by common oatp substrates. Mutual inhibition with DHEAS was also observed, suggesting that oatp2 mediates $\mathrm{E}_{1} \mathrm{~S}$ as well as DHEAS transport (Table 1).

\section{The BBB efflux transport of uremic toxins}

Indoxyl sulfate (IS), a uremic toxin, is excreted from the kidney and accumulates in uremic patients who have a reduced renal function. OAT1 and OAT3 contribute to the renal uptake of IS. ${ }^{34}$ IS is eliminated from the brain and OAT3 mediates the brain-to-blood efflux transport of IS $^{35}$ (Table 1). The brain concentration of indoxyl sulfate under normal conditions is 3.4 times lower than that in serum. ${ }^{36}$ This limited distribution could be due to the BBB efflux transport of IS mediated by OAT3 as a CNS detoxification system (FIG. 3).

\section{The BBB efflux transport of xenobiotics}

P-glycoprotein (P-gp/MDR1/ABCB1) is a well-characterized efflux transporter of xenobiotics. P-gp is a primary active transporter of relatively lipophilic compounds, such as the anticancer drug, vinblastine, cyclosporin $\mathrm{A}$, and the cardiac glycoside, digoxin, by direct consumption of ATP. There are several other types of brain-to-blood efflux transport systems for xenobiotics. The brain distribution of 6-mercaptopurine (6-MP) is limited due to its dominant brain-to-blood efflux transport $^{37}$ (FIG. 3). This limited distribution results in the penetration and proliferation of leukemic cells in the brain. Abluminally localized OAT3 is involved in the brain-to-blood efflux transport of 6-MP at the $\mathrm{BBB}^{30}$ (Table 1).

Antiviral drugs, such as AZT and DDI, are known to exhibit restricted distribution to the brain. ${ }^{38}$ Using the BEI method, these drugs were found to undergo efflux from the brain to the circulating blood across the BBB via a probenecid-sensitive carrier-mediated transport system. This is different from the nucleoside transport system because thymidine and inosine failed to inhibit AZT and DDI efflux transport, respectively. ${ }^{39}$

ATP binding cassette $(\mathrm{ABC})$ transporters are thought to play roles in excretion from the BCECs to the blood. In addition to P-gp, mRNA expression of multidrug resistance-associated protein (MRP) 1, 4, 5, and 6 has been detected in primary cultured bovine BCECs and the bovine brain capillary-enriched fraction. ${ }^{40}$ MRP1 and 5 are predominantly localized on the apical membrane fraction of primary cultured BCECs, and MRP4 is localized almost equally on the apical and basolateral membrane fractions. ${ }^{41}$ However, the localization of these subtypes in BCECs in the in vivo brain is still unclear. The efflux rate of $E_{2} 17 \beta G$ from the brain is reduced in Mrp1 knock- 
out mice, whereas the efflux rate is unchanged in Eisai hyperbilirubinemic rats (EHBR; Mrp2-deficient mutant rat). ${ }^{42}$ Therefore, Mrp1 contributes in part to the efflux transport of $\mathrm{E}_{2} 17 \beta \mathrm{G}$ at the BBB (Table 1).

ATP binding cassette transporter family $\mathrm{G}$ member 2 (ABCG2) is localized on the luminal membrane of human and rat BCECs. ${ }^{13,43}$ Dimer formation has been reported to be necessary to express the function of ABCG2 in vitro ${ }^{44}$. We have shown that $\mathrm{ABCG} 2$ forms a disulfide-linked complex in rat BCECs, and conditionally immortalized rat BCECs exhibit ABCG2-mediated efflux transport. ${ }^{13}$ The recent report by Cisternino et al. ${ }^{45}$ showed the involvement of ABCG2 in the BBB permeability of mitoxantrone and prazosin in an inhibition study involving in situ brain perfusion (Table 1). The contribution of each $\mathrm{ABC}$ transporter subtype to the $\mathrm{BBB}$ efflux transport is an important issue that remains to be resolved.

\section{The BBB efflux transport of macromolecules}

The BBB possesses a receptor-mediated transcytosis process for some physiologically active peptides. Zhang and Pardridge ${ }^{46,47}$ used the BEI method to show that IgG and apo-transferrin, which is an iron-free transferrin, undergo efflux from brain to the circulating blood (FIG. 3 and Table 1). Rat apo-transferrin is more rapidly exported from brain to blood than holo-transferrin, which is an iron-bind transferrin.

Deposition of amyloid- $\beta$ peptides (A $\beta)$, mainly $\mathrm{A} \beta_{1-40}$ and $\mathrm{A} \beta_{1-42}$, is a pathologic feature of Alzheimer's disease (AD). Shibata et al. $^{48}$ have reported that $\mathrm{A} \beta_{1-40}$ undergoes efflux from the brain across the $\mathrm{BBB}$, and low-density lipoprotein-related protein (LRP-1) is involved in this efflux process (Table 1). In addition, Lam et al. ${ }^{49}$ have reported the direct interaction of P-gp with $\mathrm{A} \beta_{1-40}$ and $\mathrm{A} \beta_{1-42}$ (Table 1). Vogelgesang et al. ${ }^{50}$ have also reported that the deposition of $A \beta_{1-40}$ and $A \beta_{1-42}$ in AD patients is inversely correlated with $\mathrm{P}$-gp expression in the brain. Clarifying the molecular mechanism of the $\mathrm{BBB}$ efflux transport of $\mathrm{A} \beta$ peptide is of great importance for the development of novel therapeutic strategies to prevent the brain deposition of $\mathrm{A} \beta$ peptide.

Vaccination, which is the passive immunization against $\mathrm{A} \beta$ peptide, is an important therapeutic strategy for reducing the brain deposition of $\mathrm{A} \beta$ peptide and improving the symptoms of $\mathrm{AD}$. Although it is still unclear how the antibody affects the brain $\mathrm{A} \beta$ peptide, two hypotheses have been proposed. One is the opsonin effect, in which the anti-A $\beta$ peptide antibody crossing the BBB binds to brain $\mathrm{A} \beta$ peptide and activates the phagocytosis of microglia. ${ }^{51}$ However, the blood-to-brain permeability of $\mathrm{IgG}$ is known to be very limited at the BBB, and the mechanism has not been elucidated yet. The other hypothesis involves the sink theory, in which the serum anti-A $\beta$ peptide antibody enhances the efflux rate of $\mathrm{A} \beta$ peptide across the $\mathrm{BBB}$ by altering the equilibrium between brain and blood. DeMattos et al. ${ }^{52}$ have reported that peripheral administration of anti-A $\beta$ peptide antibody results in a rapid increase in plasma $\mathrm{A} \beta$ peptide and markedly reduces $A \beta$ deposition in the brain. The transport system of the BBB is likely to play a crucial role in the effect of vaccination.

\section{CONCLUSION}

Recent BBB research has succeeded in changing the functional concept of the BBB from that of a static barrier to a complex of transport systems including various efflux transport systems. Clarifying the mechanisms of the BBB efflux transport system will provides us with a detailed knowledge of the physiological function of the $\mathrm{BBB}$ in the CNS and will also allow us to develop more effective and safer CNS drugs. In particular, understanding the molecular basis of the BBB efflux transport is important for improving the efficiency of CNS drug development because BBB efflux transport is one of the major barriers to successful drug delivery. A continuing multidisciplinary approach to BBB research will markedly increase our knowledge of BBB function, thereby improving the delivery of drugs to the brain.

Acknowledgments: The authors acknowledge the great support and valuable suggestions for this work from Dr. Hideo Sugita in the National Center of Neurology and Psychiatry (Tokyo, Japan).

\section{REFERENCES}

1. Reese TS, Karnovsky MJ. Fine structural localization of bloodbrain barrier to exogenous peroxidase. J Cell Biol 34:207-217, 1967.

1a. Reese TS, Feder N, Brightman MW. Electron microscopic study of the blood-brain and blood-cerebrospinal fluid barriers with microperoxidase. J Neuropathol Exp Neurol 30:137-138, 1971.

1b. Cordon-Cardo C, O'Brien JP, Casals D, Rittman-Grauer L, Biedler JL, Melamed MR, et al. Multidrug-resistance gene (P-glycoprotein) is expressed by endothelial cells at blood-brain barrier sites. Proc Natl Acad Sci USA 86:695-698, 1989.

2. Tsuji A, Terasaki T, Takabatake Y, Tenda Y, Tamai I, Yamashima $\mathrm{T}$, et al. P-glycoprotein as the drug efflux pump in primary cultured bovine brain capillary endothelial cells. Life Sci 51:1427-1437, 1992.

3. Schinkel AH, Smit JJ, van Tellingen O, Beijnen JH, Wagenaar E, van Deemter L, et al. Disruption of the mouse mdr1a P-glycoprotein gene leads to a deficiency in the blood-brain barrier and to increased sensitivity to drugs. Cell 77:491-502, 1994.

4. Terasaki T, Hosoya K. The blood-brain barrier efflux transporters as a detoxifying system for the brain. Adv Drug Deliv Rev 36:195209, 1999.

5. Kakee A, Terasaki T, Sugiyama Y. Brain efflux index as a novel method of analyzing efflux transport at the blood-brain barrier. J Pharmacol Exp Ther 277:1550-1559, 1996.

5a. Zhang Y, Schuetz JD, Elmquist WF, Miller DW. Plasma membrane localization of multidrug resistance-associated protein (MRP) homologues in brain capillary endothelial cells. J Pharmacol Exp Ther 311:449-455, 2004.

6. Mori S, Takanaga H, Ohtsuki S, Deguchi T, Kang YS, Hosoya K, et al. Rat organic anion transporter 3 (rOAT3) is responsible for brain-to-blood efflux of homovanillic acid at the abluminal mem- 
brane of brain capillary endothelial cells. J Cereb Blood Flow Metab 23:432-440, 2003.

7. Terasaki T, Ohtsuki S, Hori S, Takanaga H, Nakashima E, Hosoya K. New approaches to in vitro models of blood-brain barrier drug transport. Drug Discov Today 8:944-954, 2003.

8. Gaillard PJ, Voorwinden LH, Nielsen JL, Ivanov A, Atsumi R, Engman $\mathrm{H}$, et al. Establishment and functional characterization of an in vitro model of the blood-brain barrier, comprising a coculture of brain capillary endothelial cells and astrocytes. Eur J Pharm Sci 12:215-222, 2001.

9. Gaillard PJ, van der Sandt IC, Voorwinden LH, Vu D, Nielsen JL, de Boer AG, et al. Astrocytes increase the functional expression of $\mathrm{P}$-glycoprotein in an in vitro model of the blood-brain barrier. Pharm Res 17:1198-1205, 2000.

10. Hosoya K, Tetsuka K, Nagase K, Tomi M, Saeki S, Ohtsuki S, et al. Conditionally immortalized brain capillary endothelial cell lines established from a transgenic mouse harboring temperature-sensitive simian virus 40 large T-antigen gene. AAPS PharmSci 2:E27, 2000.

11. Hosoya K, Takashima T, Tetsuka K, Nagura T, Ohtsuki S, Takanaga $\mathrm{H}$, et al. mRNA expression and transport characterization of conditionally immortalized rat brain capillary endothelial cell lines; a new in vitro BBB model for drug targeting. J Drug Target $8: 357-370,2000$

12. Hori S, Ohtsuki S, Hosoya K, Nakashima E, Terasaki T. A pericyte-derived angiopoietin- 1 multimeric complex induces occludin gene expression in brain capillary endothelial cells through Tie-2 activation in vitro. J Neurochem 89:503-513, 2004.

13. Hori S, Ohtsuki S, Tachikawa M, Kimura N, Kondo T, Watanabe $\mathrm{M}$, et al. Functional expression of rat ABCG2 on the luminal side of brain capillaries and its enhancement by astrocyte-derived soluble factor(s). J Neurochem 90:526-536, 2004.

14. Choi TB, Pardridge WM. Phenylalanine transport at the human blood-brain barrier. Studies with isolated human brain capillaries. J Biol Chem 261:6536-6541, 1986.

15. O'Kane RL, Martinez-Lopez I, DeJoseph MR, Vina JR, Hawkins RA. $\mathrm{Na}^{+}$-dependent glutamate transporters (EAAT1, EAAT2, and EAAT3) of the blood-brain barrier. A mechanism for glutamate removal. J Biol Chem 274:31891-31895, 1999.

16. Sanchez del Pino MM, Peterson DR, Hawkins RA. Neutral amino acid transport characterization of isolated luminal and abluminal membranes of the blood-brain barrier. J Biol Chem 270:1491314918, 1995.

17. Yamazaki M, Neway WE, Ohe T, Chen I, Rowe JF, Hochman JH, et al. In vitro substrate identification studies for p-glycoproteinmediated transport species difference and predictability of in vivo results. J Pharmacol Exp Ther 296:723-735, 2001.

18. Kakee A, Takanaga H, Terasaki T, Naito M, Tsuruo T, Sugiyama Y. Efflux of a suppressive neurotransmitter, GABA, across the blood-brain barrier. J Neurochem 79:110-118, 2001.

19. Tetsuka K, Takanaga H, Ohtsuki S, Hosoya K, Terasaki T. The L-isomer-selective transport of aspartic acid is mediated by ASCT2 at the blood-brain barrier. J Neurochem 87:891-901, 2003.

20. Takanaga H, Ohtsuki S, Hosoya K, Terasaki T. GAT2/BGT-1 as a system responsible for the transport of $\gamma$-aminobutyric acid at the mouse blood-brain barrier. J Cereb Blood Flow Metab 21:12321239,2001

21. Hardebo JE, Owman C. Characterization of the in vitro uptake of monoamines into brain microvessels. Acta Physiol Scand 108:223229, 1980.

22. Wakayama K, Ohtsuki S, Takanaga H, Hosoya K, Terasaki T. Localization of norepinephrine and serotonin transporter in mouse brain capillary endothelial cells. Neurosci Res 44:173-180, 2002.

23. Hosoya K, Sugawara M, Asaba H, Terasaki T. Blood-brain barrier produces significant efflux of L-aspartic acid but not D-aspartic acid in vivo evidence using the brain efflux index method. $\mathrm{J} \mathrm{Neu}$ rochem 73:1206-1211, 1999.

24. Betz AL, Goldstein GW. Polarity of the blood-brain barrier neutral amino acid transport into isolated brain capillaries. Science 202: 225-227, 1978.

25. Takanaga H, Tokuda N, Ohtsuki S, Hosoya K, Terasaki T. ATA2 is predominantly expressed as system $\mathrm{A}$ at the blood-brain barrier and acts as brain-to-blood efflux transport for L-proline. Mol Pharmacol 61:1289-1296, 2002.

26. Emanuelsson BM, Paalzow L, Sunzel M. Probenecid-induced accumulation of 5-hydroxyindoleacetic acid and homovanillic acid in rat brain. J Pharm Pharmacol 39:705-710, 1987.

27. Kim CS, Roe CR, Mann JD, Breese GR. Octanoic acid produces accumulation of monoamine acidic metabolites in the brain interaction with organic anion transport at the choroid plexus. $\mathrm{J} \mathrm{Neu}$ rochem 58:1499-1503, 1992.

28. Morrison PF, Morishige GM, Beagles KE, Heyes MP. Quinolinic acid is extruded from the brain by a probenecid-sensitive carrier system a quantitative analysis. J Neurochem 72:2135-2144, 1999.

29. Kusuhara H, Sekine T, Utsunomiya-Tate N, Tsuda M, Kojima R, $\mathrm{Cha} \mathrm{SH}$, et al. Molecular cloning and characterization of a new multispecific organic anion transporter from rat brain. J Biol Chem 274:13675-13680, 1999.

30. Mori S, Ohtsuki S, Takanaga H, Kikkawa T, Kang YS, Terasaki T. Organic anion transporter 3 is involved in the brain-to-blood efflux transport of thiopurine nucleobase analogs. J Neurochem 90:931941, 2004.

31. Asaba H, Hosoya K, Takanaga H, Ohtsuki S, Tamura E, Takizawa T, et al. Blood-brain barrier is involved in the efflux transport of a neuroactive steroid, dehydroepiandrosterone sulfate, via organic anion transporting polypeptide 2. J Neurochem 75:1907-1916, 2000.

32. Gao B, Stieger B, Noe B, Fritschy JM, Meier PJ. Localization of the organic anion transporting polypeptide 2 (Oatp2) in capillary endothelium and choroid plexus epithelium of rat brain. $J$ Histochem Cytochem 47:1255-1264, 1999.

33. Hosoya K, Asaba H, Terasaki T. Brain-to-blood efflux transport of estrone-3-sulfate at the blood-brain barrier in rats. Life Sci 67: 2699-2711, 2000.

34. Deguchi T, Kusuhara H, Takadate A, Endou H, Otagiri M, Sugiyama Y. Characterization of uremic toxin transport by organic anion transporters in the kidney. Kidney Int 65:162-174, 2004.

35. Ohtsuki S, Asaba H, Takanaga H, Deguchi T, Hosoya K, Otagiri $\mathrm{M}$, et al. Role of blood-brain barrier organic anion transporter 3 (OAT3) in the efflux of indoxyl sulfate, a uremic toxin its involvement in neurotransmitter metabolite clearance from the brain. J Neurochem 83:57-66, 2002.

36. Muting D. Studies on the pathogenesis of uremia. Comparative determinations of glucuronic acid, indican, free and bound phenols in the serum, cerebrospinal fluid, and urine of renal diseases with and without uremia. Clin Chim Acta 12:551-554, 1965.

37. Deguchi Y, Yokoyama Y, Sakamoto T, Hayashi H, Naito T, Yamada $S$, et al. Brain distribution of 6-mercaptopurine is regulated by the efflux transport system in the blood-brain barrier. Life Sci 66:649-662, 2000.

38. Terasaki T, Pardridge WM. Restricted transport of $3^{\prime}$-azido-3'deoxythymidine and dideoxynucleosides through the blood-brain barrier. J Infect Dis 158:630-632, 1988.

39. Takasawa K, Terasaki T, Suzuki H, Sugiyama Y. In vivo evidence for carrier-mediated efflux transport of 3'-azido-3'-deoxythymidine and $2^{\prime}, 3^{\prime}$-dideoxyinosine across the blood-brain barrier via a probenecid-sensitive transport system. J Pharmacol Exp Ther 281: 369-375, 1997.

40. Zhang Y, Han H, Elmquist WF, Miller DW. Expression of various multidrug resistance-associated protein (MRP) homologues in brain microvessel endothelial cells. Brain Res 876:148-153, 2000.

41. Zhang Y, Schuetz JD, Elmquist WF, Miller DW. Plasma membrane localization of multidrug resistance-associated protein (MRP) homologues in brain capillary endothelial cells. J Pharmacol Exp Ther 311:449-455, 2004.

42. Sugiyama D, Kusuhara H, Lee YJ, Sugiyama Y. Involvement of multidrug resistance associated protein 1 (Mrp1) in the efflux transport of $17 \beta$ estradiol-D-17 $\beta$-glucuronide (E217 $\beta$ G) across the blood-brain barrier. Pharm Res 20:1394-1400, 2003.

43. Cooray HC, Blackmore CG, Maskell L, Barrand MA. Localisation of breast cancer resistance protein in microvessel endothelium of human brain. Neuroreport 13:2059-2063, 2002.

44. Kage K, Tsukahara S, Sugiyama T, Asada S, Ishikawa E, Tsuruo $\mathrm{T}$, et al. Dominant-negative inhibition of breast cancer resistance protein as drug efflux pump through the inhibition of S-S dependent homodimerization. Int J Cancer 97:626-630, 2002. 
45. Cisternino S, Mercier C, Bourasset F, Roux F, Scherrmann JM. Expression, up-regulation, and transport activity of the multidrugresistance protein Abcg2 at the mouse blood-brain barrier. Cancer Res 64:3296-3301, 2004.

46. Zhang Y, Pardridge WM. Mediated efflux of $\mathrm{IgG}$ molecules from brain to blood across the blood-brain barrier. J Neuroimmunol 114:168-172, 2001.

47. Zhang Y, Pardridge WM. Rapid transferrin efflux from brain to blood across the blood-brain barrier. J Neurochem 76:1597-1600, 2001.

48. Shibata M, Yamada S, Kumar SR, Calero M, Bading J, Frangione $\mathrm{B}$, et al. Clearance of Alzheimer's amyloid-ss(1-40) peptide from brain by LDL receptor-related protein-1 at the blood-brain barrier. J Clin Invest 106:1489-1499, 2000.

49. Lam FC, Liu R, Lu P, Shapiro AB, Renoir JM, Sharom FJ, et al. $\beta$-Amyloid efflux mediated by p-glycoprotein. J Neurochem 76 : 1121-1128, 2001.
50. Vogelgesang S, Cascorbi I, Schroeder E, Pahnke J, Kroemer HK, Siegmund W, et al. Deposition of Alzheimer's $\beta$-amyloid is inversely correlated with P-glycoprotein expression in the brains of elderly non-demented humans. Pharmacogenetics 12:535-541, 2002.

51. Bard F, Cannon C, Barbour R, Burke RL, Games D, Grajeda H, et al. Peripherally administered antibodies against amyloid $\beta$-peptide enter the central nervous system and reduce pathology in a mouse model of Alzheimer disease. Nat Med 6:916-919, 2000.

52. DeMattos RB, Bales KR, Cummins DJ, Dodart JC, Paul SM, Holtzman DM. Peripheral anti-A $\beta$ antibody alters CNS and plasma A $\beta$ clearance and decreases brain $\mathrm{A} \beta$ burden in a mouse model of Alzheimer's disease. Proc Natl Acad Sci USA 98:8850-8855, 2001.

53. Terasaki T, Hosoya K. The brain efflux index method (BEI). In: Alfred Benzon Symposium 45, Brain Barrier Systems (Palson OB, Knudsen GM, Moos T, eds). Cophenhagen, Denmark: Munksgaad, 1998. 